EPJ Web of Conferences 109,07002 (2016)

DOI: $10.1051 /$ epjconf/201610907002

(C) Owned by the authors, published by EDP Sciences, 2016

\title{
Shedding Light on the EOS-Gravity Degeneracy and Constrain- ing the Nuclear Symmetry Energy from the Gravitational Binding Energy of Neutron Stars
}

\author{
Xiao-Tao He $\mathrm{H}^{1,2, \mathrm{a}}$, F. J. Fattoyever,3,b , Bao-An Li ${ }^{2, \mathrm{c}}$, and W. G. Newton ${ }^{2, \mathrm{~d}}$ \\ ${ }^{1}$ College of Material Science and Technology, Nanjing University of Aeronautics and Astronautics, Nanjing \\ 210016, China \\ ${ }^{2}$ Department of Physics and Astronomy, Texas A\&M University-Commerce, Commerce, TX 75429, USA \\ ${ }^{3}$ Center for Exploration of Energy and Matter, Indiana University, Bloomington, IN 47408, USA
}

\begin{abstract}
A thorough understanding of properties of neutron stars requires both a reliable knowledge of the equation of state (EOS) of super-dense nuclear matter and the strong-field gravity theories simultaneously. To provide information that may help break this EOS-gravity degeneracy, we investigate effects of nuclear symmetry energy on the gravitational binding energy of neutron stars within GR and the scalar-tensor subset of alternative gravity models. We focus on effects of the slope $L$ of nuclear symmetry energy at saturation density and the high-density behavior of nuclear symmetry energy. We find that the variation of either the density slope $L$ or the high-density behavior of nuclear symmetry energy leads to large changes in the binding energy of neutron stars. The difference in predictions using the GR and the scalar-tensor theory appears only for massive neutron stars, and even then is significantly smaller than the difference resulting from variations in the symmetry energy.
\end{abstract}

\section{Introduction}

Neutron stars (NSs) are among the most compact, dense and neutron-rich objects known in the Universe. The typical surface compactness parameter of NSs is of the order of $\eta_{R} \equiv 2 G M / R c^{2} \approx 0.4$, which is 5 orders of magnitudes larger than that at the surface of the sun $\left(\eta_{\odot} \equiv 2 G M_{\odot} / R_{\odot} c^{2} \approx\right.$ $\left.4 \times 10^{-6}\right)$. If one uses instead a more natural measure of the gravitational field strength, the "curvature" parameter $\xi \equiv 2 G M / c^{2} r^{3}$, which is related to the non-vanishing components of the Riemann tensor in vacuum $\mathcal{R}^{1}{ }_{010}=-\xi$ as well as to the Kretschmann invariant $\mathcal{K}=2 \sqrt{3} \xi$ outside the star, the surface curvature for a $1.4 M_{\odot}$ neutron star is then 14 orders of magnitudes larger than that at the surface of the Sun, where Solar System tests of General Relativity in the weak field regime are usually performed. The strong gravitational field makes NSs as good testbeds for the untested strong-field gravity regime, while their high-density matter content make them as natural laboratories for determining the EOS of the super-dense nuclear matter [1-5]. However, it cannot simultaneously be good

\footnotetext{
ae-mail: hext@nuaa.edu.cn

be-mail: ffattoye@indiana.edu

ce-mail: bao-an.li@tamuc.edu

de-mail: william.newton@tamuc.edu
} 
testing ground for both. There is a possible degeneracy between the models of the neutron-star matter EOSs and models of gravity applied to describe their properties. How to break this degeneracy is a longstanding problem to which many recent studies have been devoted (see e.g., [4-18]). This talk is based on the work originally published in Ref. [19]. Here we present our analyses of the extent to which a EOS-Gravity degeneracy exists when models of gravity are limited to classical GR and Scalar-Tensor (ST) theories, while variations of the EOS appear as a result of variation of the slope $L$ of the nuclear symmetry energy at saturation density or the high-density behavior of the nuclear symmetry energy. We find that the variation of either the density slope $L$ or the high-density behavior of nuclear symmetry energy within their uncertainty ranges lead to significant changes in the binding energy of NSs. In particular, the variations are significantly greater than those that result from ST theories of gravity, leading to the conclusion that within those subset of gravity models, measurements of neutron star properties constrain mainly the EOS. Further investigations demonstrate that only EOSs with the soft symmetry energy at high-density are consistent with constraints on the gravitational binding energy of PSR J0737-3039B.

\section{The Equation of State of Nuclear Matter}

In general, the EOS of neutron-rich nucleonic matter can be expressed in terms of the binding energy per nucleon in asymmetric nuclear matter of isospin asymmetry $\alpha=\left(\rho_{n}-\rho_{p}\right) / \rho$ as $E(\rho, \alpha)=E_{0}(\rho)+$ $\mathcal{S}(\rho) \alpha^{2}+\mathcal{O}\left(\alpha^{4}\right)$, where $E_{0}(\rho)$ is the binding energy per nucleon in symmetric nuclear matter $(\alpha=0)$, $\mathcal{S}(\rho)$ is the nuclear symmetry energy, and $\rho=\rho_{n}+\rho_{p}$ is the total baryon density with $\rho_{n}\left(\rho_{p}\right)$ being the neutron (proton) density. The EOS can further be characterized in terms of bulk nuclear properties by expanding both $E_{0}(\rho)$ and $\mathcal{S}(\rho)$ in Taylor series around nuclear saturation density $\rho_{0}=0.16 \mathrm{fm}^{-3}$, i.e., $E_{0}(\rho)=B_{0}+\frac{1}{2} K_{0} \chi^{2}+O\left(\chi^{3}\right)$ and $\mathcal{S}(\rho)=J+L \chi+\frac{1}{2} K_{\text {sym }} \chi^{2}+O\left(\chi^{3}\right)$, where $\chi \equiv\left(\rho-\rho_{0}\right) / 3 \rho_{0}$ quantifies the deviations of the nuclear density from its saturation value. Terrestrial experimental nuclear data such as the ground state properties of finite nuclei and energies of giant resonances tightly constrain the binding energy at saturation $B_{0}$ and the nuclear incompressibility coefficient $K_{0}$, hence constrain the EOS of symmetric nuclear matter $E_{0}(\rho)$. Whereas the symmetry energy at saturation $J$ is more or less known, its density slope $L$ is largely unconstrained, and the overall behavior of the symmetry energy at supra-saturation densities is quite uncertain.

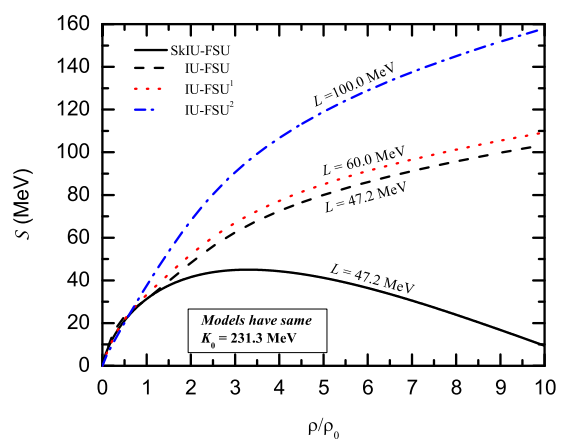

Figure 1. (color online). Density dependence of the nuclear symmetry energy: the solid black line corresponds to the symmetry energy predictions in SkIU-FSU, the dashed black line corresponds to the original IU-FSU model, the dotted red and dash-dotted blue lines are symmetry energy predictions in the IU-FSU-like models with density slope $L=60.0 \mathrm{MeV}$ $\left(\mathrm{IU}_{-\mathrm{FSU}}{ }^{1}\right.$ ) and $L=100.0 \mathrm{MeV}\left(\mathrm{IU}-\mathrm{FSU}^{2}\right.$ ), respectively. Figure is taken from Ref. [19].

For our base models we use two recently established EOSs for neutron-rich nucleonic matter within the IU-FSU Relativistic Mean Field (RMF) and the SkIU-FSU Skyrme-Hartree-Fork (SHF) models [20-22]. The slope of the symmetry energy at saturation for these models is $L=47.2 \mathrm{MeV}$. While the density dependence of symmetry energy $\mathcal{S}(\rho)$ at subsaturation densities is almost identical 
for these models, at supra-saturation densities of $\gtrsim 1.5 \rho_{0}$ the two models give significantly different predictions of $\mathcal{S}(\rho)$. To test the sensitivity of the gravitational binding energy of neutron stars to the variations of properties of neutron-rich nuclear matter around saturation density, we have further introduced two RMF models with density slopes of the symmetry energy at saturation density of $L=60 \mathrm{MeV}$ and $L=100 \mathrm{MeV}$. Shown in Fig. 1 are the density dependence of the symmetry energy for these four models [19].

\section{Comparison of Predictions between GR \& Scalar-Tensor Theory Using Different EOSs}

Properties of neutron stars are not only sensitive to the underlying EOS, but also to the strong-field behavior of gravity. We will consider neutron stars in both GR and in the simplest natural extension of the GR known as the scalar-tensor theory of gravity. According to this theory, in addition to the second-rank metric tensor, $g_{\mu \nu}$, the gravitational force is also mediated by a scalar field, $\varphi$. The action defining this theory can be written in the most general form as [23, 24]

$$
S=\frac{c^{4}}{16 \pi G} \int d^{4} x \sqrt{-g^{*}}\left[R^{*}-2 g^{* \mu \nu} \partial_{\mu} \varphi \partial_{\nu} \varphi-V(\varphi)\right]+S_{\text {matter }}\left(\psi_{\text {matter }} ; A^{2}(\varphi) g_{\mu \nu}^{*}\right),
$$

where $R^{*}$ is the Ricci scalar curvature with respect to the so-called Einstein frame metric $g_{\mu \nu}^{*}$ and $V(\varphi)$ is the scalar field potential. The physical metric is defined as $g_{\mu \nu} \equiv A^{2}(\varphi) g_{\mu \nu}^{*}$. The relativistic equations for stellar structure in hydrostatic equilibrium can be written as

$$
\begin{aligned}
\frac{d M_{\mathrm{G}}(r)}{d r} & =\frac{4 \pi}{c^{2}} r^{2} \mathcal{E}(r) A^{4}(\varphi)+\frac{r^{2}}{2}\left(1-\frac{2 G M(r)}{c^{2} r}\right) \chi^{2}(r)+\frac{r^{2}}{4} V(\varphi), \\
\frac{d M_{\mathrm{B}}(r)}{d r} & =4 \pi m_{\mathrm{B}} r^{2} \rho(r) A^{3}(\varphi), \\
\frac{d \varphi(r)}{d r} & =\chi(r), \\
\frac{d \chi(r)}{d r} & =\left[1-\frac{2 G M(r)}{c^{2} r}\right]^{-1}\left\{\frac{4 \pi G}{c^{4}} A^{4}(\varphi)[\alpha(\varphi)(\mathcal{E}(r)-3 P(r))+r \chi(r)(\mathcal{E}(r)-P(r))]\right. \\
& \left.-\frac{2}{r}\left(1-\frac{G M(r)}{c^{2} r}\right) \chi(r)+\frac{1}{2} r \chi(r) V(\varphi)+\frac{1}{4} \frac{d V(\varphi)}{d \varphi}\right\}, \\
& =-(\mathcal{E}(r)+P(r))\left[1-\frac{2 G M(r)}{c^{2} r}\right]^{-1}\left\{\frac{4 \pi G}{c^{4}} r A^{4}(\varphi) P(r)+\frac{G}{c^{2} r^{2}} M(r)\right. \\
& \left.\left(1-\frac{2 G M(r)}{c^{2} r}\right)\left(\frac{1}{2} r \chi^{2}(r)+\alpha(\varphi) \chi(r)\right)-\frac{1}{4} r V(\varphi)\right\},
\end{aligned}
$$

where $\alpha(\varphi) \equiv \partial \ln A(\varphi) / \partial \varphi$. Following the Ref. [23] we set $V(\varphi)=0$ which can only appear in models of modified gravity, and we consider a coupling function of the form $A(\varphi)=\exp \left(\alpha_{0} \varphi+\frac{1}{2} \beta_{0} \varphi^{2}\right)$ [19]. For a given central pressure $P(0)=P_{\mathrm{c}}$ one can integrate the equations above from the center of the star to $r \rightarrow \infty$, where the only input required is the EOS of dense matter in chemical equilibrium. At the center of the star the Einstein frame boundary conditions are given as $P(0)=P_{\mathrm{c}}, \mathcal{E}(0)=$ $\mathcal{E}(\mathrm{c}), \varphi(0)=\varphi_{\mathrm{c}}, \chi(0)=0$, while at infinity we demand cosmologically flat solution to agree with the observation $\lim _{r \rightarrow \infty} \varphi(r)=0$. The stellar coordinate radius is determined by the condition of $P\left(r_{\mathrm{s}}\right)=0$. The physical radius of a neutron star is found in the Jordan frame as $R_{\mathrm{NS}}=A^{2}\left[\varphi\left(r_{\mathrm{s}}\right)\right] r_{\mathrm{s}}$. 
Notice however that the physical stellar mass as measured by an observer at infinity matches with the coordinate mass, since at infinity the coupling function approaches unity. According to the latest observational constraints the quadratic parameter of the scalar-tensor theory should take values of not smaller than $\beta_{0} \gtrsim-5.0$ Ref. [23, 25]. Similarly the absolute value of the linear parameter is also constrained very well by observation. We will therefore choose the upper bounds on these parameters as $\alpha_{0}^{2}<2.0 \times 10^{-5}$ and $\beta_{0}>-5.0$ [25].

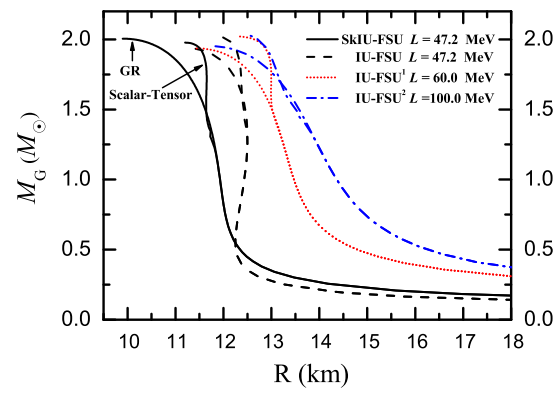

Figure 2. (color online). The mass-versus-radius relation of neutron stars calculated using EOSs considered in this work. For the scalar-tensor theory the upper observational bound on parameters $\left\{\alpha_{0}, \beta_{0}\right\}=\left\{\sqrt{2.0 \times 10^{-5}},-5.0\right\}$ have been used. Figure is taken from Ref. [19].

In Fig. 2 we present our results for the mass versus radius relation of neutron stars as calculated by using the four EOSs discussed above both within GR and the scalar-tensor theory with $\left\{\alpha_{0}, \beta_{0}\right\}=$ $\left\{\sqrt{2.0 \times 10^{-5}},-5.0\right\}$. we observe a larger radius in the scalar-tensor theories than in GR only for massive neutron stars. Even so, the changes in radii are much smaller than those that arise from variation of the stiffness of the symmetry energy. In general, differences in predictions using the GR and the scalar-tensor theories within current observational bound on their parameters are much smaller than those due to the uncertainties in the EOS.

\section{Constraining the Nuclear Symmetry Energy from the Gravitational Binding Energy}

The fractional gravitational binding energy $\mathcal{B} \equiv M_{\mathrm{G}}-M_{\mathrm{B}}$ [26] [See Eqns.(2)-(3)] as a function of the total gravitational mass of a neutron star is displayed in Fig. 3. Notice that in general the GR predictions give a lower absolute value of the fractional binding energy for a given NS mass then the scalar-tensor theory. However the difference is quite negligible compared to the uncertainties coming from the variations of the EOS. Furthermore, all low-mass NSs are indistinguishable for an observer in these two models of gravity, because the critical value for the so-called "spontaneous scalarizaton" is reached only when NSs masses exceed about 1.4 solar mass. Thus measurements of NS mass and radii will lead to significantly constrain density dependence of the symmetry energy, rather than constraining gravity models within the GR and scalar-tensor theories.

As both the mass-verus-radius relation and gravitational binding energy of NSs are more sensitive to different EOSs, we further examine the effects on binding energy of the uncertain symmetry energy in more details (see again Fig. 3). Notice that with the very similar low-density symmetry energy up to about $1.5 \rho_{0}$, but different high-density behaviors, predictions for the $\mathcal{B} / M_{\mathrm{G}}$ in the SkIU-FSU and IU-FSU models are quite different. This effect becomes more evident with the increase of the total gravitational mass. With the IU-FSU as a reference baseline model then the relative changes in the fractional gravitational binding energies are $5.32 \%, 6.52 \%$ and $9.89 \%$ for $1.25 M_{\odot}, 1.4 M_{\odot}$ and $1.9 M_{\odot}$ neutron stars respectively. This is in contrast to the predictions of the binding energy in models with soft and stiff symmetry energies at saturation-e.g., the IU-FSU with $L=47.2 \mathrm{MeV}$ and the 


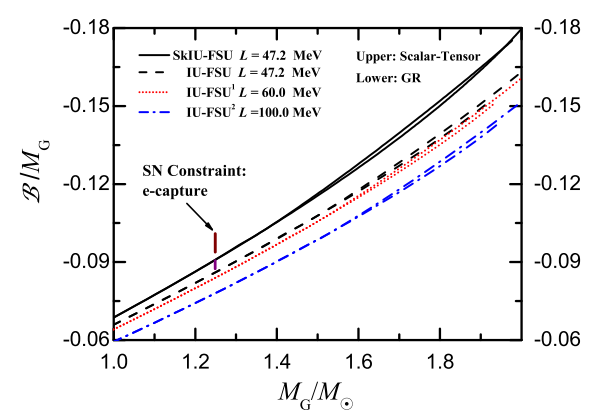

Figure 3. (color online). The fractional gravitational binding energy versus the gravitational mass of a neutron star for a set of the EOSs discussed in the text. The constraints on the gravitational binding energy of PSR J0737-3039B, under the assumption that it is formed in an electron-capture supernova, are given by the vertical brown and purple lines. Figure is taken from Ref. [19] with slight modifications.

IU-FSU ${ }^{2}$ with $L=100 \mathrm{MeV}$. Again using the former as a reference model, then the relative changes in the fractional gravitational binding energies are $-10.26 \%,-8.85 \%$ and $-7.44 \%$ for $1.25 M_{\odot}, 1.4 M_{\odot}$ and $1.9 M_{\odot}$ neutron stars respectively. Hence the fractional binding energy is more sensitive to the saturation density slope of the symmetry energy for low-mass neutron stars [27], while more sensitive to the supra-saturation behavior of the symmetry energy for massive stars [19].

The gravitational mass of the lighter pulsar PSR J0737-3039B is determined very accurately to be $M_{\mathrm{G}}=1.2489 \pm 0.0007 M_{\odot}$ [28], which is one of the lowest reliably measured mass for any neutron star up to date. Due to its low mass it was suggested that this NS might have been formed as a result of the collapse of an electron-capture supernova from a an O-Ne-Mg white dwarf progenitor [29]. Under this assumption, it is estimated that the baryonic mass of the precollapse O-Ne-Mg core should lie between $1.366 M_{\odot}<M_{\mathrm{B}}<1.375 M_{\odot}$ [29] and $1.358 M_{\odot}<M_{\mathrm{B}}<1.362 M_{\odot}$ [30]. In Fig. 3 we show that only models with the soft symmetry energy are consistent with these set of constraints.

\section{Summary}

Interpreting properties of neutron stars require a resolution in the degeneracy between the EOS for super-dense matter and the strong-field gravity. With the goal of providing information that may help break this degeneracy we have studied effects of the nuclear symmetry energy within its current uncertain range on the mass-versus-radius relation and the binding energy of NSs within both the GR and the scalar-tensor theory of gravity. We have found that radii of neutron stars are primarily sensitive to the underlying EOS through the density dependence of the symmetry energy. Within the simplest natural extension of the GR known as the scalar-tensor theory of gravity, and by using upper observational bounds on the parameters of the theory, we found a negligible change in the binding energy of NSs over the whole mass range, and significant changes in radii only for NSs whose mass are well above $1.4 M_{\odot}$. Even then, the changes in radii are found to be much smaller than those that result from variation of the stiffness of the symmetry energy. We have also shown that the gravitational binding energy is moderately sensitive to the nuclear symmetry energy, with lower mass neutron stars $\lesssim 1.4 M_{\odot}$ probing primarily the stiffness of the symmetry energy at nuclear saturation density, and massive stars being more sensitive to the high density behavior of the symmetry energy. A combination of observational and theoretical arguments on the gravitational binding energy of PSR J0737-3039B imply that only EOSs with soft symmetry energy at high density are consistent with the extracted values of the binding energy. 


\section{Acknowledgements}

This work is supported in part by the National Natural Science Foundation of China under Grant No. 11275098, 11275067 and 11320101004, the US National Science Foundation under Grant No. PHY-1068022 and the CUSTIPEN (China-U.S. Theory Institute for Physics with Exotic Nuclei) under DOE grant number DE-FG02-13ER42025, and DOE grants DE-SC0013702 (Texas A\&M UniversityCommerce), DE-FG02-87ER40365 (Indiana University) and DE-SC0008808 (NUCLEI SciDAC Collaboration).

\section{References}

[1] J.M. Lattimer, M. Prakash, Phys. Rept. 442, 109 (2007).

[2] J.M. Lattimer, Ann. Rev. Nucl. Part. Sci. 62, 485 (2012).

[3] B.A. Li, L.W. Chen, C.M. Ko, Phys. Rept. 464, 113 (2008).

[4] P. Pani, E. Berti, V. Cardoso, J. Read, Phys. Rev. D84, 104035 (2011).

[5] K.Y. Eksi, C. Gungor, M.M. Turkouglu, Phys. Rev. D89, 063003 (2014).

[6] C.M. Will, Living Rev. Rel. 9, 3 (2006).

[7] T. Harada, Phys. Rev. D57, 4802 (1998).

[8] H. Sotani, K.D. Kokkotas, Phys. Rev. D70, 084026 (2004).

[9] P.D. Lasky, H. Sotani, D. Giannios, Phys. Rev. D78, 104019 (2008).

[10] D.H. Wen, B.A. Li, L.W. Chen, Phys. Rev. Lett. 103, 211102 (2009).

[11] A. Cooney, S. DeDeo, D. Psaltis, Phys. Rev. D82, 064033 (2010).

[12] M. Horbatsch, C. Burgess, JCAP 1108, 027 (2011).

[13] A.S. Arapoglu, C. Deliduman, K.Y. Eksi, JCAP 1107, 020 (2011).

[14] C. Deliduman, K. Eksi, V. Keles, JCAP 1205, 036 (2012).

[15] H. Sotani, Phys. Rev. D86, 124036 (2012).

[16] W. Lin, B.A. Li, L.W. Chen, D.H. Wen, J. Xu, J. Phys. G41, 075203 (2014).

[17] K. Yagi, L.C. Stein, N. Yunes, T. Tanaka, Phys.Rev. D87, 084058 (2013).

[18] H. Sotani, Phys. Rev. D89, 104005 (2014).

[19] X.T. He, F.J. Fattoyev, B.A. Li, W.G. Newton, Phys. Rev. C91, 015810 (2015).

[20] F.J. Fattoyev, C.J. Horowitz, J. Piekarewicz, G. Shen, Phys. Rev. C82, 055803 (2010).

[21] F.J. Fattoyev, W.G. Newton, J. Xu, B.A. Li, Phys. Rev. C86, 025804 (2012).

[22] F.J. Fattoyev, J. Carvajal, W. Newton, B.A. Li, Phys.Rev. C87, 015806 (2013).

[23] T. Damour, G. Esposito-Farese, Phys.Rev. D54, 1474 (1996).

[24] S.S. Yazadjiev, D.D. Doneva, K.D. Kokkotas, K.V. Staykov, JCAP 1406, 003 (2014).

[25] P.C. Freire, N. Wex, G. Esposito-Farese, J.P. Verbiest, M. Bailes et al., Mon.Not.Roy.Astron.Soc. 423, 3328 (2012).

[26] S. Weinberg, Gravitation and Cosmology (John Wiley \& Sons, New York, 1972)

[27] W. Newton, B.A. Li, Phys.Rev. C80, 065809 (2009).

[28] M. Kramer, I.H. Stairs, R. Manchester, M. McLaughlin, A. Lyne et al., Science 314, 97 (2006).

[29] P. Podsiadlowski, J. Dewi, P. Lesaffre, J. Miller, W. Newton et al., Mon.Not.Roy.Astron.Soc. 361, 1243 (2005).

[30] F. Kitaura, H.T. Janka, W. Hillebrandt, Astron.Astrophys. 450, 345 (2006). 